\title{
REFERENCES
}

1. M. Herzberger, Strahlenoptik, Springer, Berlin, 1931.

2. M. Herzberger, Zur Optik inhomogener. Mittel, Zeitschrift für Instrumentenkunde, 53, 436-443 (1933).

3. J. I. Singe, Geometrical optics, Cambridge Tracts No. 37, 1937.

4. R. K. Luneberg, Mathematical theory of optics, Brown University mimeographed lecture notes, Providence, R. I., 1944.

5. P. Franklin, .Ycthods of advanced calculus, McGraw-Hill Book Co., New York, 1944.

6. S. Gorn, Rays in isotropic, non-homogeneous media, Air Force Technical Report No. 6262 (1951).

\section{ON SUPERSONIC FLOW PAST AN OSCILLATING WEDGE*}

\section{By MILTON D. VAN DYKE (Ames Aeronautical Laboratory, Moffett Field, Calif.)}

1. Introduction. In order to study the limitations of the linearized theory of oscillating airfoils, Carrier ${ }^{1}$ has analyzed supersonic flow past a thick wedge which oscillates slightly about its apex. Considerably greater insight into the nonlinear effects of thickness is gained by generalizing the analysis to include other locations of the pivot.

2. Analysis. Consider a wedge fixed in a supersonic stream at a Mach number above that at which the shock wave detaches from the apex. If now the wedge executes slight oscillations, the shock will remain attached, and the flow field can be found by superposing a linearized acoustic field upon the nonlinear steady flow. For harmonic oscillations of frequency $a_{2} c$, Carrier has shown ${ }^{1,2}$ that the velocity components and pressure downstream of the shock are given by

$$
\begin{aligned}
& u=U_{2}+a_{2}\left(\varphi_{x}+E_{y}\right), \quad v=a_{2}\left(\varphi_{y}-E_{x}\right), \\
& p=p_{2}\left[1-\gamma\left(\varphi_{\imath}+M \varphi_{x}\right)\right],
\end{aligned}
$$

where

$$
\begin{aligned}
& \varphi=e^{i c\left(t-M x / \beta^{2}\right)} \sum^{\infty}\left(a_{\nu} \cosh \nu \theta+b_{\nu} \sinh \nu \theta\right) J_{\nu}(k r), \\
& E=e^{i r\left(t-x / M-\lambda_{y} / M \beta^{2}\right)} \sum^{\infty} c_{\nu} J_{\nu}(k \xi y),
\end{aligned}
$$

and the deflection of the shock wave from its steady position is

$$
\psi=\left(1-\rho_{1} / \rho_{2}\right)^{-1} e^{i c\left(t-\lambda . M_{v} / \beta^{2}\right)} \sum^{\infty} d_{\nu} J_{v}(k \xi y) .
$$

Here, $U_{2}, a_{2}, M, p_{2}$ and $\rho_{2}$ are the flow speed, sound speed, Mach number, pressure and density downstream of the shock in steady flow (and $\rho_{1}$ the density upstream), $\gamma$ is the adiabatic exponent, $t$ the time multiplied by $a_{2}, \beta^{2}=M^{2}-1, k=c / \beta^{2}, \tanh \theta=$ $\beta y / x, r^{2}=x^{2}-\beta^{2} y^{2}$ and $\xi^{2}=1+\lambda^{2}-M^{2}$. The coordinate system and $\lambda$ are defined in Fig. 1.

*Received Feb. 18, 1953.

${ }^{1} \mathrm{G}$. F. Carrier, The oscillating wedge in a supersonic stream, J. Aer. Sci., 16, 150-152 (1949).

${ }^{2} \mathrm{G}$. F. Carrier, On the stability of the supersonic flows past a wedge, Q. Appl. Math. 6, 356-378 (1949). 
The pertinent jump conditions across the shock wave, which can be imposed at the steady shock position, were shown to be equivalent to

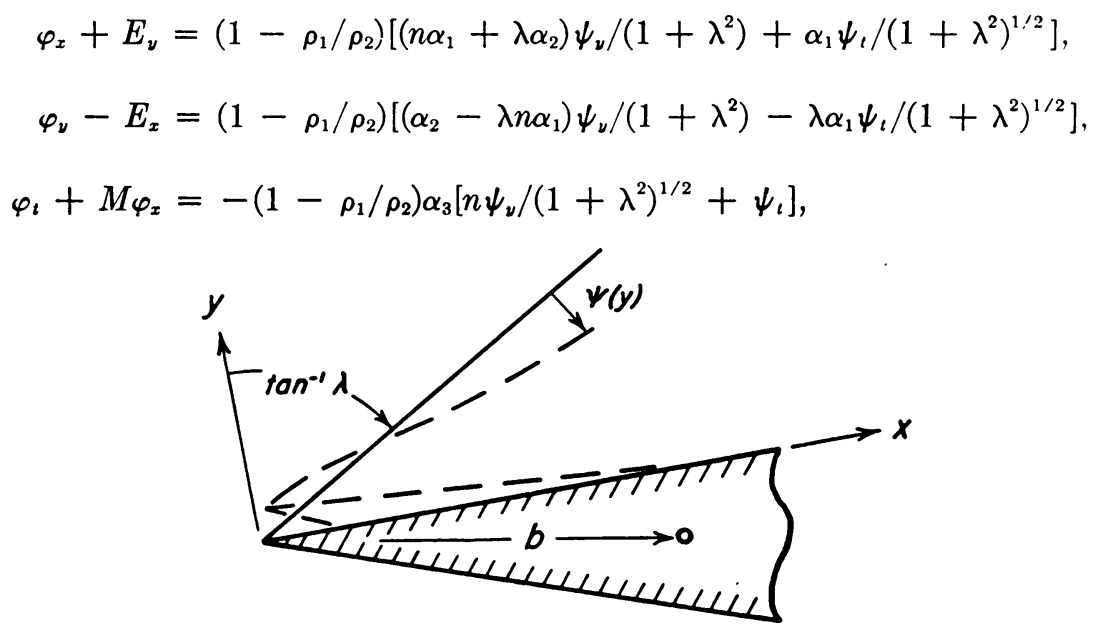

Fig. 1. Coordinate system.

where $\alpha_{1}=\left(2+4 m^{2}\right) / 3\left(1-m^{2}\right), \alpha_{2}=\left(5+m^{2}\right) / 6 m$ and $\alpha_{3}=-m\left(5+m^{2}\right) / 3\left(1-m^{2}\right)$ for $\gamma=7 / 5$. Here $m$ and $n$ are the components of $M$ normal and tangential to the undisturbed shock.

Suppose now that the wedge is pivoted at a point a distance $b$ downstream of its apex, and oscillates with small angle of attack $\alpha=R e\left(e^{i c t}\right)$ in some unit system. The boundary condition of tangent flow at the surface is ${ }^{3}$

$$
\left(\varphi_{\nu}-E_{x}\right)_{\nu=0}=-[M+i c(x-b \cos \epsilon)] e^{i c t},
$$

where $\epsilon$ is the semi-vertex angle of the wedge. Following Carrier, using the generating function for the Bessel coefficients we find that for $\nu \geq 1$ the $b_{\nu}$ are given by

$$
b_{\nu}=i \nu\left[\tau^{\nu}+(-\tau)^{-\nu}\right] / \beta k+b \cos \epsilon\left[\tau^{\nu}-(-\tau)^{-\nu}\right],
$$

where $\tau=i(M+\beta)$.

For the special case of rotation about the apex, the summations in Eqs. (3) to (5) begin with $\nu=1$. For other pivot positions, however, a term $\nu=0$ must be added to Eq. (5) to account for the fact that as the apex of the wedge oscillates about the origin the attached shock wave moves with it. The question of whether corresponding modifcations are required in the series for $\varphi$ and $E$ can be answered as follows. Consider the pivot point to be moved indefinitely far downstream, and the amplitude of angular oscillation correspondingly reduced, so that ultimately the wedge simply executes a small vertical translational ("plunging") oscillation. Furthermore, let the frequency of oscillation tend to zero. In the limit, the wedge stands fixed and slightly above and parallel with its original position. It is clear that in this steady flow the shock wave is displaced from its original position, but the velocity perturbations associated with $\varphi$ and $E$ are zero. Hence a $\nu=0$ mode must be added only to Eq. (5).

${ }^{3}$ Here an error in the tangency condition in the reference of footnote 1 has been corrected. Furthermore, numerous typographical errors in the subsequent equations of that paper have been rectified. 
The additional coefficient thus introduced is determined by the condition that the shock wave must at all times meet the apex of the wedge, which gives

$$
d_{0}=-b\left(1-\rho_{1} / \rho_{2}\right)\left(1+\lambda^{2}\right)^{-1 / 2}(\lambda \cos \epsilon-\sin \epsilon) .
$$

Then the shock wave conditions yield three recurrence relations for determining successively the coefficients $a_{\nu}, c_{\nu}$ and $d_{v}(\nu \geq 1)$ in terms of $d_{0}$ and the $b_{\nu}$. In matrix form, with $\tanh \theta_{0}=\beta / \lambda$, these are

$$
\begin{gathered}
\left(\begin{array}{ccc}
\cosh \nu \theta_{0} & \xi & -n \sqrt{1-m^{2}}\left(m \alpha_{1}+\alpha_{2}\right) / M \\
\beta \sinh \nu \theta_{0} & 0 & \sqrt{1-m^{2}}\left(n^{2} \alpha_{1}-m \alpha_{2}\right) / M \\
-M \cosh \nu \theta_{0} & 0 & -n \sqrt{1-m^{2}} \alpha_{3}
\end{array}\right]\left[\begin{array}{l}
a_{v+1} \\
c_{v+1} \\
d_{v+1}
\end{array}\right] \\
\left.+\left(\begin{array}{ccc}
-\cosh \nu \theta_{0} & -\xi & n \sqrt{1-m^{2}}\left(m \alpha_{1}+\alpha_{2}\right) / M \\
\beta \sinh \nu \theta_{0} & 0 & -\sqrt{1-m^{2}}\left(n^{2} \alpha_{1}-m \alpha_{2}\right) / M \\
M \cosh \nu \theta_{0} & 0 & n \sqrt{1-m^{2}} \alpha_{3}
\end{array}\right]\right)\left[\begin{array}{l}
a_{v-1} \\
c_{v-1} \\
d_{v-1} b u t 2 d_{0}
\end{array}\right) \\
+\left(\begin{array}{ccc}
-2 i M \cosh \nu \theta_{0} & -2 i \lambda / M & 2 i\left[m\left(1-m^{2}\right) \alpha_{1}+n^{2} \alpha_{2}\right] / M \\
0 & 2 i \beta^{2} / M & -2 i n\left[\left(1-m^{2}\right) \alpha_{1}-m \alpha_{2}\right] / M \\
2 i \cosh \nu \theta_{0} & 0 & 2 i\left(1-m^{2}\right) \alpha_{3} \\
\sinh \nu \theta_{0} & -\sinh \nu \theta_{0} & \left.-2 i M \sinh \nu \theta_{0}\right) \\
\beta \cosh \nu \theta_{0} & \beta \cosh \nu \theta_{0} & 0
\end{array}\right)\left[\begin{array}{l}
a_{v} \\
c_{v} \\
d_{v}
\end{array}\right) \\
+\left(\begin{array}{ccc}
b_{v+1} \\
b_{v-1} \\
b_{v}
\end{array}\right]=0,
\end{gathered}
$$

for $\nu=0,1 \cdots$, where it is understood that the $a_{v}, b$, and $c_{v}$ vanish for $\nu<1$, and the $d_{v}$ for $\nu<0$. Note that for $\nu=1, d_{v-1}$ is to be replaced by $2 d_{0}$.

3. Example: Slow oscillations. The solution can be readily converted into an expansion in powers of frequency. Then retaining only linear terms in frequency shows that for slow oscillations the surface pressure coefficient, referred to conditions upstream of the shock, is

$$
C_{p} \equiv\left(p-p_{1}\right) / \frac{1}{2} \rho_{1} U_{1}^{2}=\bar{C}_{p}-\left(2 / M_{1}^{2}+\gamma \bar{C}_{p}\right)\left[A \alpha+(B b \cos \epsilon+C x) \alpha^{*} / a_{2}\right] .
$$

Here $\bar{C}_{\nabla}$ is the value for steady flow, $M_{1}$ the free-stream Mach number, and $\alpha$ and $\alpha$. the instantaneous angle of attack and its (true) time derivative. The coefficients $A, B, C$ are

$$
\begin{gathered}
A=-M^{2} n \alpha_{3} / \mu \quad B=M(n-m \tan \epsilon) \alpha_{3} / \mu \\
C=M \frac{2 \mu-2 M^{2} \lambda n \alpha_{3} / \beta^{2}+\left[\left(M^{2}+n^{2}\right) \alpha_{2}-m n^{2}\right] \alpha_{3} / \mu}{\lambda \mu-\beta^{2} n \alpha_{3}}+\frac{M\left(M^{2}+1\right) n \alpha_{3}}{\beta^{2} \mu}
\end{gathered}
$$


where $\mu=n^{2} \alpha_{1}-m \alpha_{2}$. For a wedge airfoil with flat base (on which the unknown base pressure is assumed to be constant, though possibly time dependent) the normal force and pitching moment coefficients are

$C_{N}=\left(2 / M_{1}^{2}+\gamma \bar{C}_{p}\right)\left[2 A \alpha+(2 B \sigma+C) \alpha \cdot \sec \epsilon / a_{2}\right]$,

$C_{M}=\left(2 / M_{1}^{2}+\gamma \bar{C}_{p}\right) \sec ^{2} \epsilon\left[A(2 \sigma-1) \alpha+\left\{B \sigma(2 \sigma-1)+C\left(\sigma-\frac{2}{3}\right)\right\} \alpha^{\cdot} \sec \epsilon^{\prime} a_{2}\right]$,

where $\sigma=c \cos ^{2} \epsilon / c$.

The term in Eq. (16) proportional to $\alpha$ represents the aerodynamic damping moment for slow oscillations, which tends to stabilize if it is negative. The boundary of neutral stability as it depends upon pivot position and free-stream Mach number is shown in Fig. 2 for a wedge airfoil of $5^{\circ}$ semi-vertex angle. Also shown are the corresponding

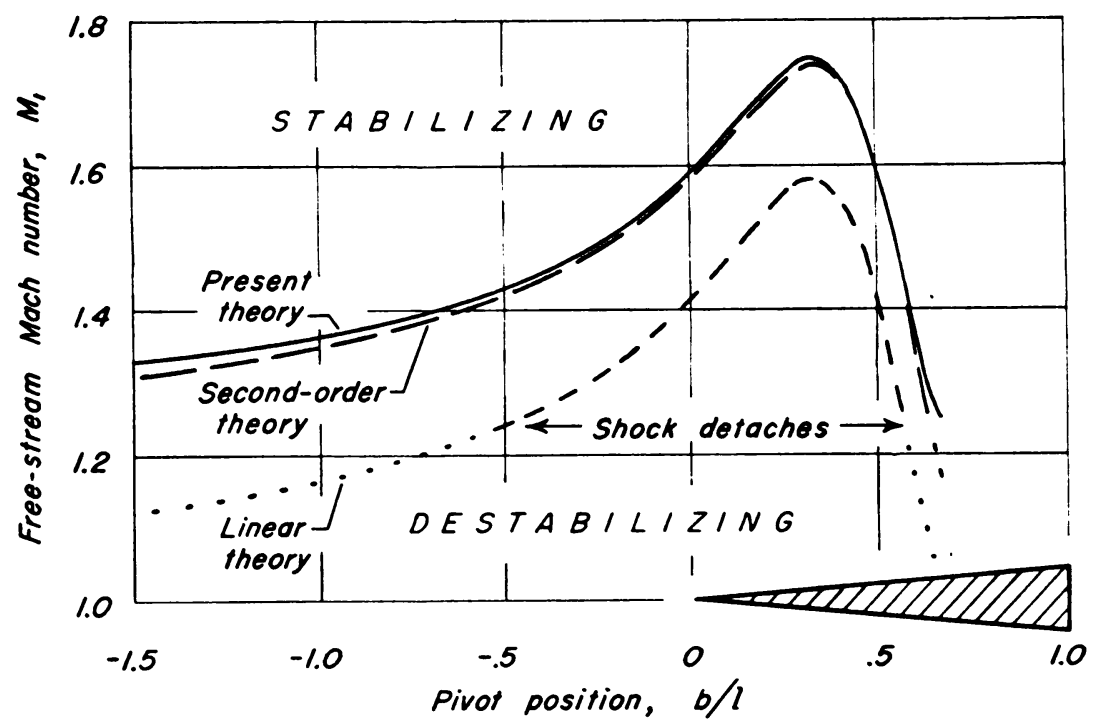

FIG. 2. Boundary of neutral stability for slowly oscillating wedge airfoil of $5^{\circ}$ semi-vertex angle.

results from linear and second-order theory ${ }^{4}$ (with which the present theory agrees when expanded in powers of $\epsilon$ ), which are applicable to any airfoil shape.

\section{A NOTE ON SUBSONIC EDGES IN UNSTEADY SUPERSONIC FLOW*}

\section{By JOHN W. MILES (University of California, Los Angeles)}

Summary. The pressure distribution due to the unsteady motion of a wing having a supersonic leading edge and a subsonic trailing edge is determined by applying a Lorentz transformation to the corresponding result for a rectangular wing. This result, valid as

${ }^{4}$ Milton D. Van Dyke, On second-order supersonic flow past a slowly oscillating airfoil, J. Aero. Sci. 20, 61 (1953).

*Received March 2, 1953. 